\title{
Medien und Suizid: der aktuelle Forschungsstand zum Werther- und Papageno-Effekt - eine Übersichtsarbeit
}

\author{
Benedikt Till · Thomas Niederkrotenthaler
}

Online publiziert: 18. November 2019

(C) Der/die Autor(en) 2019

\begin{abstract}
Zusammenfassung Suizidales Verhalten wird durch Suiziddarstellungen in den Massenmedien beeinflusst. In zahlreichen Studien konnte gezeigt werden, dass sensationsträchtige Darstellungen von Suizid in den Medien zu Imitationssuiziden führen. Dieses Phänomen ist in der Wissenschaft auch unter dem Begriff „Werther-Effekt“ bekannt. In einer Reihe von Ländern wurden daher von Expert_Innen für Suizidprävention Medienempfehlungen zur Berichterstattung über Suizid entwickelt, um die Qualität der Berichterstattung über Suizid zu verbessern und in weiterer Folge Imitationssuizide zu verhindern. Nicht alle Darstellungen von Suizid in den Medien sind dabei schädlich. In mehreren Studien konnte gezeigt werden, dass Medienberichte, die auf Personen fokussieren, die sich in einer suizidalen Krise befunden haben, aber in der Lage waren, diese Krise zu bewältigen, zu einem Rückgang an Suizidalität bei Rezipient_Innen führen. In Anlehung an Papageno, den Protagonisten in Mozarts Oper Die Zauberflöte, der seine suizidale Krise bewältigt, wird dieser protektive Effekt in der wissenschaftlichen Literatur auch als „Papageno-Ef-
\end{abstract}

Teile dieser Übersichtsarbeit wurden in abgewandelter Form und englischer Sprache veröffentlicht in De Leo \& Postuvan (Eds.) (2019), Reducing the Toll of Suicide: Resources for

Communities, Groups, and Individuals. Hogrefe Publishing. www.hogrefe.com, in Niederkrotenthaler \& Stack (Eds.) (2017), Media \& suicide: International perspectives on research, theory, \& policy. Transaction Books, sowie in der Zeitschrift Suicidologi (https://www.med.uio.no/klinmed/ english/research/centres/nssf/resources/the-journalsuicidologi/) (Niederkrotenthaler und Till, in Druck).

\section{B. Till $(\bowtie) \cdot T$. Niederkrotenthaler}

Unit Suizidforschung \& Mental Health Promotion,

Abteilung für Sozial- und Präventivmedizin, Zentrum für

Public Health, Medizinische Universität Wien,

Kinderspitalgasse 15, 1090 Wien, Österreich

benedikt.till@meduniwien.ac.at fekt“ bezeichnet. In dieser Übersichtsarbeit werden der gegenwärtige Forschungsstand und die rezentesten Studien zum Werther- und Papageno-Effekt diskutiert. Diese Befunde können Aufschluss darüber geben, wie effektive Medienkampagnen zur Suizidprävention gestaltet sein müssen, um eine möglichst protektive Wirkung ohne Risiko eines schädlichen Effekts zu erzielen. Da die psychologischen Mechanismen des Papageno-Effekts nach wie vor nicht vollständig erforscht sind, sind weitere Untersuchungen zur protektiven Wirkung von Medienberichten über Krisenbewältigungen notwendig, insbesondere Studien mit klinischen Populationen oder anderen Risikogruppen.

Schlüsselwörter Suizid · Medien · Werther-Effekt . Papageno-Effekt

\section{Media and suicide: current state of research on Werther and Papageno effect-a literature review}

Summary Suicide portrayals in the mass media are one of the factors that influence suicidal behavior. There is a considerable amount of evidence demonstrating that sensationalist portrayals of suicide in the media trigger imitational suicides. This effect is commonly referred to as Werther effect. In many countries, experts for suicide prevention have developed media recommendations for suicide reporting in order to improve the quality of suicide reporting and prevent imitational suicides. Not all suicide portrayals in the media are harmful. In fact, media stories focusing on individuals who have experienced a suicidal crisis, but were able to successfully overcome their crisis, have been shown to reduce suicidality in some audiences. Based on Papageno's mastered crisis in Mozart's Magic Flute, this protective effect was 
named Papageno effect. In this literature review, we discuss the current state of research and review the most recent studies on Werther and Papageno effect. These recent findings may inform media awareness campaigns for suicide prevention and help them to balance risks and opportuntities. With psychological mechanisms of the Papageno effect being still unclear, more research on the protective effects of media stories of coping with suicidal crises are warranted, particularly studies involving clinical populations or other high-risk groups.

Keywords Suicide $\cdot$ Media $\cdot$ Werther effect · Papageno effect

\section{Einleitung}

Suizid ist niemals auf einen enzigen Grund zurückzuführen, vielmehr entwickelt sich Suizidalität aus einer multikausalen Genese, an der unterschiedliche biologische, soziale, psychologische und kulturelle Faktoren beteiligt sind (Mann et al. 2005). Ein Faktor, der hierbei eine wichtige Rolle spielt, ist die Darstellung von Suizid und Suizidalität in den Medien (Niederkrotenthaler et al. 2014; Sisask und Värnik 2012; WHO 2017). Hierbei ist allerdings entscheidend, auf welche Art und Weise Suizidalität dargestellt wird und wer die Rezipient_Innen dieser Darstellungen sind (Till und Niederkrotenthaler 2018).

\section{Sensationsträchtige Darstellungen von Suizid in den Medien: der Werther-Effekt}

Mehr als 150 wissenschaftliche Untersuchungen haben gezeigt, dass sensationsträchtige Darstellungen von Suizid in Nachrichtenberichten aufgrund von Imitationssuiziden zu einem Anstieg der Suizidrate in der Bevölkerung führen (Niederkrotenthaler et al. 2014; Sisask und Värnik 2012; WHO 2017). Dieser Effekt wird in der wissenschaftlichen Literatur auch als WertherEffekt bezeichnet, in Anlehung an eine angebliche Reihe von Imitationssuiziden nach der Veröffentlichung von Goethes berühmten Werk Die Leiden des jungen Werther im Jahr 1774, welches mit dem Suizid des Protagonisten endet (Niederkrotenthaler et al. 2007). Der Werther-Effekt konnte seit seiner erstmaligen Beschreibung durch Phillips (1974) mittlerweile in verschiedensten Kulturräumen repliziert werden (WHO 2017). So zeigte zum Beispiel eine kürzlich erschienene Studie von Fink et al. (2018), dass es nach der sensationsträchtigen Nachrichtenberichterstattung über den Suizid des berühmten Schauspielers Robin Williams in den USA zu einem Anstieg der Suizidrate um fast $10 \% \mathrm{kam}$.

Der Werther-Effekt ist keineswegs auf Suiziddarstellungen in Nachrichtenmedien beschränkt, sondern kann auch bei Darstellungen von Suizid in fiktionalen Filmen und TV-Serien auftreten. So konnte beispielsweise nach der Austrahlung der sechsteiligen
Fernsehreihe Tod eine Schülers, in der der Eisenbahnsuizid eines 19-jährigen Schülers dargestellt wird, ein Anstieg an Eisenbahnsuiziden unter Jugendlichen in Deutschland festgestellt werden (Schmidtke und Häfner 1986). Gould und Shaffer (1986) verglichen die Anzahl der Suizide sowie Suizidversuche in den zwei Wochen vor der Ausstrahlung von Filmen, die mit dem Suizid der jeweiligen Hauptfigur endeten, mit jenen in den zwei Wochen danach und fanden einen Anstieg an Suiziden und Suizidversuchen im Großraum New York. In einer randomisierten, kontrollierten Studie berichteten Zuschauer_Innen, die einen Suizidalitätsscore über dem Median der Stichprobe aufwiesen, einen Anstieg an Suizidgedanken nach dem Sehen eines Hollywood-Films, der mit dem Suizid der Protagonistin endete (Till et al. 2015). Hinweise auf einen Werther-Effekt zeigten sich auch nach der Ausstrahlung der ersten Staffel der NetflixSerie Tote Mädchen lügen nicht, die mit dem Suizid der Protagonistin endete. Aufgrund der expliziten Darstellung des Suizids wurden in mehreren Ländern unmittelbar nach dem Release Empfehlungen zum Umgang mit der Serie in Schulen herausgegeben (Arendt et al. 2017; Till und Niederkrotenthaler 2018). Ressourcen zum Umgang mit der Serie können zum Beispiel unter folgender Webadresse abgerufen werden: https://www.13reasonswhytoolkit.org. Mehrere kürzlich erschienene Studien konnten einen Anstieg an Suiziden und Suizidversuchen unter Jugendlichen in den USA nach der Ausstrahlung der Serie aufzeigen (z. B. Bridge et al. im Druck; Cooper et al. 2018; Niederkrotenthaler et al. 2019). Dieser war insbesondere unter Mädchen im Alter von 10-19 Jahren ausgeprägt - jene Gruppe, die dem Rollenmodell in der Serie am ähnlichsten war (Niederkrotenthaler et al. 2019).

Die psychologischen Mechanismen, die diesem Effekt zugrunde liegen, sind bis heute noch nicht hinreichend bekannt (Till und Niederkrotenthaler 2018). Auf Basis des Konzepts des prä-suizidalen Syndroms von Erwin Ringel (1976) geht man davon aus, dass Menschen in der Zeit vor einem Suizid oder einem Suizidversuch den Suizid häufig als eine gedankliche Möglichkeit in Betracht ziehen und dabei zwischen lebenserhaltenden und selbstzerstörerischen Impulsen schwanken. Gerade in diese Phase der Ambivalenz können Botschaften der Umwelt, insbesondere Medienbotschaften, den Ausgang dieses inneren Konflikts beeinflussen (Till und Niederkrotenthaler 2014b). Andere Autoren (z. B. Blood und Pirkis 2001) verweisen in diesem Zusammenhang auf das so genannte Lernen am Modell nach Bandura (1977). Diesem Konzept zufolge eignet sich der Mensch seine Verhaltensweisen u.a. anhand von Lernprozessen an, die durch die Beobachtung anderer Personen ausgelöst werden (Bandura 1977). Hierzu gehört auch suizidales Verhalten von Menschen in Mediendarstellungen (Blood und Pirkis 2001). Auch soziale Identifikationsprozesse wurden in der wissenschaftlichen Literatur mit dem Werther-Effekt in Verbindung ge- 
bracht (Arendt et al. 2016; Fu und Yip 2009; Stack 1992). Dementsprechend konnte in eine Studie aus Österreich ein höheres Risiko für einen Imitationseffekt festgestellt werden, wenn sich Rezipient_In und jene Person, über deren Suizid in der Zeitung berichtet wurde, hinsichtlich soziodemographischer Variablen ähnlich waren (Niederkrotenthaler et al. 2009). Ebenso war in einer randomisierten, kontrollierten Studie der Anstieg an Suizidalität nach dem Sehen eines Films, der mit dem Suizid der Protagonistin endete, besonders stark, wenn sich die Zuschauer_Innen mit vergleichsweise höherer Suizidalität mit der suizidalen Protagonistin identifizieren konnten (Till et al. 2015). Dies weist darauf hin, dass Identifikation mit der jeweiligen Medienfigur ein wichtiges Element für den Werther-Effekt darstellt. Auch das Aumaß an Berichterstattung ist von Bedeutung. So ist zum Beispiel die mehrfache Berichterstattung über einen Suizid in Zeitungen mit einem Anstieg an Suiziden in der Bevölkerung assoziiert (vgl. z. B. Niederkrotenthaler et al. 2010a).

\section{Medienempfehlungen zur Berichterstattung über Suizid}

Um Imitationssuizide $\mathrm{zu}$ vermeiden und eine verantwortungsvolle Berichterstatung über Suizid $\mathrm{zu}$ fördern, ist die Zusammenarbeit mit Medien eine wichtige Komponente der Suizidprävention (Till und Niederkrotenthaler 2014b). Dies zeigt zum Beispiel die Erfahrung mit den Suiziden in der Wiener U-Bahn: Mit dem Ausbau des U-Bahn-Systems in Wien Mitte der 1980-er Jahre kam es zu einem starken Anstieg der Suizide und Suizidversuche in U-Bahn-Stationen. Der Anstieg diese Suizidmethode wurde insbesondere auf die häufige und sensationsträchtige Berichterstattung über diese Vorfälle in den Medien zurückgeführt. Aus diesem Grund wurden Medienempfehlungen zur Berichterstattung über Suizid entwickelt und Journalist_Innen im Rahmen einer Medienkampagne vorgestellt. Die Empfehlungen, welche im Sinne einer freiwilligen Selbstkontrolle implementiert wurden, legten zum Beispiel nahe, keine Details über den jeweiligen Suizid zu berichten, sensationsträchtige Sprache und vereinfachende Erklärungen für den Suizid zu vermeiden, Alternativen zu Suizid aufzuzeigen und Kontaktadressen von Hilfseinrichtungen zu nennen. Aufgrund der Implementierung der Medienempfehlungen kam es zu einem Rückgang an sensationsträchtiger Berichterstattung über Suizide in Österreich, insbesondere über U-Bahn-Suizide, und einer Senkung der Zahl an Suiziden und Suizidversuchen in der Wiener U-Bahn um mehr als $70 \%$, welche seither auf diesem Niveau verblieb (Etzersdorfer und Sonneck 1998; Etzersdorfer et al. 1992; Niederkrotenthaler und Sonneck 2007; Till und Niederkrotenthaler 2014b).

Dieses Beispiel macht nicht nur deutlich, dass sensationsträchtige Berichterstattung über Suizid Imita- tionssuizide auslöst, sondern auch dass dieser negative Effekt durch verantwortungsvolle Medienberichterstattung verhindert werden kann. Mittlerweile wurden in vielen Ländern ähnliche Mediemempfehlungen zur Berichterstattung über Suizid entwickelt (Pirkis et al. 2006) und auch von der WHO (2017) heraus- und weitergegeben. Positive Erfahrungen im Sinne einer verbesserten Qualität in der Berichterstattung über Suizid oder einer Senkung der Suizidrate konnten nicht nur in Österreich, sondern auch in der Schweiz, in Australien sowie in Hong Kong gemacht werden (Michel et al. 2000; Pirkis et al. 2009; Sisask und Värnik 2012). In Anbetracht der vielen Länder, die Medienempfehlungen nutzen, ist die Zahl an Studien über die Effektivität von Medienempfehlungen insgesamt jedoch nach wie vor gering (Niederkrotenthaler et al. 2014). Auch für Suiziddarstellungen in fiktionalen Filmen oder TV-Serien wurden mittlerweile Empfehlungen entwickelt und herausgegeben, so etwa von Mindframe (2007) in Australien, den Samaritans (2017) in Großbritannien oder der National Action Alliance for Suicide Prevention (2019) in den USA. Darüber hinaus gibt es seit kurzem in den USA auch Empfehlungen zur Berichterstattung über sogenannte „Mass Shootings“ (Suicide Awareness Voices of Education 2017), denen ebenfalls ein Imitationseffekt zugrunde zu liegen scheint (Jetter 2017). Bisher gibt es aber noch keine Studien, in denen die Wirksamkeit solcher Empfehlungen evaluiert wurde.

Ein spezifisches und sehr wichtiges Element von den Medienempfehlungen zur Berichterstattung über Suizid ist für gewöhnlich die Empfehlung zur Verwendung neutraler Sprache, wenn über Suizid geschrieben wird. So wird zum Beispiel im Österreichischen Leitfaden zur Berichterstattung über Suizid empfohlen, Formulierungen wie „erfolgreicher, nicht erfolgreicher oder missglückter Selbstmord“ zu vermeiden und durch Beschreibungen wie etwa „starb durch Suizid“ oder „tötete sich“ zu ersetzen (Tomandl et al. 2019). Auch von pathologisierenden und kriminalisierenden Entwertungen wie der Begriff „Selbstmord“ oder auch von befürwortenden Billigungen, die sich in Begriffen wie „Freitod“ manifestieren, wird abgeraten und an ihrer Stelle der neutrale Begriff „Suizid“ empfohlen (Tomandl et al. 2019). Aus rationaler Sicht könnte man annehmen, dass derartige Formulierungen austauschbar wären, da sie ähnliche Sachinformationen beinhalten. Jedoch weisen beide Formulierungen unterschiedliche assoziative Bedeutungen auf, sodass hierbei unterschiedliche Kognitionen und Konzepte bei den Rezipient_Innen aktiviert werden, was in der Sozialpsychologie als Framing-Effekt bezeichnet wird (Kahneman 2011). In einer kürzlich durchgeführten randomisierten, kontrollierten Studie konnten entsprechende Framing-Effekte für die Begriffe „Suizid“, „Selbstmord“ und „Freitod“ nachgewiesen werden. Die Teilnehmer_Innen der Studie lasen in allen Interventionsgruppen dieselben drei Artikel über Suizid, nur der jeweilige in den Artikeln 
verwendete Begriff für Suizid („Suizid“, „Selbstmord“ oder „Freitod“) variierte zwischen den Gruppen. Die Ergebnisse zeigten, dass die unterschiedliche Rahmung der Nachrichtenartikel sowohl die Wortwahl der Teilnehmer_Innen bei der schriftlichen Zusammenfassung der Artikel als auch deren Einstellung zu Suizid bei Menschen mit unheilbaren Erkrankungen beeinflusste. So war die Akzeptanz von Suizid bei unheilbar kranken Menschen bei jenen Teilnehmer_Innen größer, die die Artikel mit dem Wort „Freitod“ gelesen hatten (Arendt et al. 2018b). Dies zeigt, dass nur ein einziges Wort in der Berichterstattung über Suizid einen Unterschied machen kann, und unterstreicht die Wichtigkeit der Empfehlung von neutraler Sprache.

Eine weitere wichtige Empfehlung betrifft die Aufklärung über Mythen und Fehlurteile über Suizid: Die Medien sollten keine Mythen verbreiten, sondern zur Aufklärung über Suizid beitragen (Tomandl et al. 2019). Medien, die zum Beispiel traditionellerweise mit Mythen über Suizid in Verbindung gebracht werden, sind Boulevardzeitungen (Schütte 2008). Till und Kollegen konnten in einer Studie zeigen, dass der Konsum von Boulevardzeitungen mit Glaube an Suizidmythen, stigmatisierende Einstellungen gegenüber suizidalen Personen und geringem Wissen über Suizid und Suizidprävention assoziiert, dieser Zusammenhang aber nicht alleine auf Alter und Bildung der Befragten zurückzuführen war (Till et al. 2018). Dies unterstreicht, dass Aufklärung über Suizid eine wichtige Komponente der Suizidprävention darstellt.

\section{Protektive Darstellungen von Suizidalität in den Medien: der Papageno-Effekt}

Für lange Zeit lag in Forschung und Präventionspraxis der Fokus fast ausschließlich auf den Risiken von Suizidberichterstattung, was zu einer Tabuisierung des Themas in der Gesellschaft beigetragen hat. Für eine Entstigmatisierung von Suizidalität bedarf es aber auch einer Behandlung dieses Themas in den Medien (Hawton und Williams 2002; Niederkrotenthaler et al. 2010a, 2014; Till und Niederkrotenthaler 2014b). Dass Berichterstattung über Suizid ohne schädliche Wirkung möglich ist, zeigte zum Beispiel eine Studie aus Österreich, in der alle Beiträge über Suizid in den 11 größten Zeitungen von Jänner bis Juni 2005 gesammelt und analysiert wurden (Niederkrotenthaler et al. 2010a): Während sensationsträchtige Zeitungsberichte über Suizid (ca. 20\%) ganz im Sinne des Werther-Effekts mit einem Anstieg an Suiziden in der Bevölkerung assoziiert waren, beinhalteten ca. $72 \%$ der gefundenen Artikel keine sensationsträchtigen Eigenschaften und waren auch nicht mit der Suizidrate assoziiert. Es stellte sich jedoch die Frage, ob Suizidberichterstattung auch einen protektiven Effekt haben könnte. In der Tat fanden Niederkrotenthaler und Kollegen, dass Berichte, die auf die Bewältigung von suizidalen Krisen fokussierten - das waren in etwas
$8 \%$ der gefundenen Artikel - mit einem Rückgang der Suizidrate assoziiert waren (Niederkrotenthaler et al. 2010a). In Anlehnung an Mozarts Oper Die Zauberflöte, in der der Protagonist, Papageno, eine suizidale Krise durchlebt, diese aber mit Hilfe von den „drei Knaben“ erfolgreich bewältigt, wird dieser protektive Effekt Papageno-Effekt genannt (Niederkrotenthaler et al. 2010a, 2010b). Dies zeigt, dass Medien einen wichtigen Beitrag im öffentlichen Bewusstsein leisten können, indem eine psychische Erkrankung nicht weiter als schicksalshafte Krankheit ohne Veränderungsmöglichkeiten dargestellt wird, sondern als eine zeitlich begrenzte Phase der Verzweiflung, die mit konkreter Hilfe bewältigt werden kann (Till und Niederkrotenthaler 2014b; Tomandl et al. 2019). Auch zeigt der Papageno-Effekt neue Wege in der Suizidprävention auf, zum Beispiel in der Gestaltung von effektiven Aufklärungskampagnen über Suizid mittels Einbeziehung von (Geschichten über) Menschen mit persönlicher Erfahrung mit der Bewältigung von suizidalen Krisen (Niederkrotenthaler et al. 2014).

\section{Neue Untersuchungen zum Papageno-Effekt}

Bei der ursprünglichen Studie zum Papageno-Effekt (Niederkrotenthaler et al. 2010a) handelte es sich um eine epidemiologische Studie, in der Effekte anhand aggregierter Daten untesucht wurden. Untersuchungen mit solchen Studiendesigns sind wichtig, unterliegen allerdings der Limitation, dass nicht sichergestellt werden kann, dass der beobachtete Effekt tatsächlich auf den jeweiligen Medieninput zurückzuführen ist. So lässt sich hierbei bespielsweise nicht feststellen, ob jene Personen, die einen Rückgang an Suizidalität wahrgenommen haben, die vermeintlich protektiven Medienberichte überhaupt rezipiert haben. Dort, wo dies möglich und ethisch vertretbar ist, sollten daher epidemiologische Studien mit Ergebnissen von Untersuchungen mit individuellen Daten (randomisierte, kontrollierte Studien bzw. Laborexperimente) ergänzt werden (Blood und Pirkis 2001; Pirkis und Blood 2001a, 2001b; Niederkrotenthaler et al. 2014; Pouliot et al. 2011). Ein Nachteil solcher Studien ist jedoch, dass hierbei nicht Suizid als Ergebnisvariable untersucht werden kann, sondern bestenfalls Risikofaktoren für Suizid, wie zum Beispiel Suizidgedanken, Depressivität oder Hoffnungslosigkeit (Pirkis und Blood 2001a, 2001b). Darüber hinaus ist nach wie vor nicht vollständig geklärt, durch welche Eigenschaften eines Artikels der Papageno-Effekt genau ausgelöst wird, welchen psychologischen Mechanismen der Effekt zugrunde liegt und welche Gruppen an Rezipient_Innen hiervon profitieren. Randomisierte, kontrollierte Studien könnten einen wichtigen Beitrag zur Erforschung dieses Effekts beitragen (Niederkrotenthaler et al. 2014).

In einer randomisierten, kontrollierten Studie mit 112 Studierenden zeigte sich zum Beispiel, dass sich Suizidgedanken, die mit dem Implicit Association Test 
(IAT; Nock et al. 2010) gemessen wurden, reduzierten, wenn die Proband_Innen zuvor einen Zeitungsartikel gelesen hatten, in dem beschrieben war, wie ein Protagonist seine suizidale Krise durch die Inanspruchnahme einer Hilfseinrichtung erfolgreich bewältigt hatte. Dieser Effekt, welcher mit der Annahme eines Papageno-Effekts konsistent ist, trat allerdings nur bei jenen Proband_Innen auf, die sich in nur einem geringen Ausmaß mit der spezifischen Problemsituation des Protagonisten identifizierten (Arendt et al. 2016). Dieses Ergebnis könnte auf einen sogenannten „Kontrastierungseffekt“ (Till et al. 2010) zurückzuführen sein: Menschen vergleichen sich laufend mit den Protagonisten in medialen Darstellungen. Da die Teilnehmer_Innen selbst nicht suizidal waren, sich hier aber mit einer (ehemals) suizidalen Person hinsichtlich ihrer suizidalen Krise verglichen, liegt es nahe, dass viele Teilnehmer_Innen zu dem Schluss kamen, dass es ihnen „ganz anders“ und besser erging als dem Protagonisten in der Geschichte (vgl. Till et al. 2010). Durch einen derartigen Vergleich würde geringere Identifikation mit dem Protagonisten somit mit einer Verringerung der Suizidalität einhergehen (Arendt et al. 2016). Es bleibt die Frage offen, ob suizidale Menschen ebenso mit einem „Kontrastierungseffekt“ reagieren würden (Till et al. 2010) - diese waren von der Studie allerdings ausgeschlossen bzw. waren nicht repräsentiert (Arendt et al. 2016). In einer weiteren Studie konnte ein Anstieg an Wissen über Suizidprävention und eine vermehrte Bereitschaft, Menschen in suizidalen Krisen $\mathrm{zu}$ helfen, festgestellt werden, nachdem Proband_Innen einen Zeitungsartikel gelesen hatten, in dem über gängige Mythen über Suizid aufgeklärt wurde und ein Protagonist die erfolgreiche Bewältigung seiner suizidaler Krise beschrieben hat (Arendt et al. 2018a).

Seit einigen Jahren werden zunehmend die Erfahrungen von Menschen, die selbst suizidale Krisen durchlebt und bewältigt haben, in der Entwicklung von Strategien und Ressourcen zur Suizidprävention einbezogen (National Action Alliance for Suicide Prevention 2014). Auf Basis dieser neuen Entwicklung wurde in einer randomisierten, kontrollierten Studie (Till et al. 2019) untersucht, ob und inwieweit auch Expert_Innen, die selbst schon einmal Suizidgedanken hatten und ihre suizidale Krise bewältigt haben, ihre persönliche Erfahrung einbringen und offen darüber erzählen können und wie hilfreich das für die Prävention sein kann. Die Teilnehmer_Innen in den beiden Interventionsgruppen lasen jeweils einen Nachrichtenartikel, in dem eine Expertin im Rahmen eines Interviews über Suizid und Suizidprävention Aufklärungsarbeit geleistet und mehrere häufig in der Gesellschaft vorkommende Mythen über Suizidalität entkräftet hat. Der einzige Unterschied zwischen den beiden Gruppen war, dass in einer Gruppe die interviewte Expertin persönliche Erfahrung mit eigener Suizidalität offenbart hat, währed dies in der anderen Interventionsgruppe nicht der Fall war. Die Ergebnis- se der Studie zeigten, dass Proband_Innen in beiden Interventionsgruppen einen Anstieg an Wissen über Suizidprävention und einer Reduktion von Suizidgedanken berichteten (Till et al. 2019). Damit konnte der Papageno-Effekt neuerlich repliziert werden. Die Studie zeigte auch, dass Aufklärung durch Suizidexpert_Innen mit persönlicher Erfahrung mit eigener Suizidalität gleichermaßen effektiv ist wie durch jene, die keine solchen Erfahrungen teilten (Till et al. 2019).

Die protektive Wirkung von Medieninhalten, die auf die Bewältigung suizidaler Krisen fokussieren, ist aber keineswegs auf Print-Medien beschränkt. So konnte in einer randomisierten, kontrollierten Studie festgestellt werden, dass sich die Lebenszufriedenheit der Studienteilnehmer_Innen verbesserte, wenn diese einen Hollywood-Film sahen, der mit der Bewältigung einer suizidalen Krise und einem Happy-End endete. Dieser Effekt war bei Personen mit Suizidalität über dem Median der Stichprobe besonders stark ausgeprägt (Till et al. 2015). Auf Basis des Papageno-Effekts haben australische Forscher_Innen in Kooperation mit professionellen Filmemacher_Innen eine dreiteilige Dokumentation mit dem Title Man Up produziert. Die Dokumentation zielt darauf ab, insbesondere Männer mit Suizidgedanken anzusprechen und beschäftigte sich mit der Frage, wie die Gesellschaft das Eigenbild von Männern und deren Verhaltensweisen formt und somit ihre psychische Befindlichkeit beeinflusst. Die Dokumentation war ein großer Erfolg, auch vom wissenschaftlichen Standpunkt aus. In mehreren Studien mit unterschiedlichen Designs konnte ein positiver Effekt auf das Hilfesuchverhalten der Männer und deren Einstellung $\mathrm{zu}$ professioneller Hilfe festgestellt werden. Suizidalität wurde nur als Nebenzielgröße untersucht und zeigte keine signifikante Veränderung (King et al. 2018a, 2018b; Schlichthorst et al. 2018).

Auch für Aufklärungsmaterial zur Suizidprävention im Internet ist der Papageno-Effekt relevant. Das Internet ist ein sehr heterogenes Medium und beinhaltet eine große Bandbreite an suizid-relevanter Information (Till und Niederkrotenthaler 2014a, 2014b). Zu den häufigsten in Suchmaschinen vorzufindenden Typen an Webseiten über Suizid zählen edukative Webseiten, die darauf abzielen, die Allgemeinbevölkerung, insbesondere Jugendliche und junge Erwachsene, über Suizidalität aufzuklären (Till und Niederkrotenthaler 2014a; Till et al. 2017). Diese Webseiten beinhalten für gewöhnlich, neben epidemiologischen Zahlen und Fakten über Suizid, Kontaktadressen zu Hilfseinrichtungen, aber auch Erzählungen von Menschen, die über persönliche Erfahrungen mit Suizidalität berichten und schildern, wie sie schwierige Phasen in ihrem Leben überstanden haben und was ihnen dabei geholfen hat (Till et al. 2017). In einer kürzlich durchgeführten randomisierten, kontrollierten Studie konnte nun die Wirksamkeit solcher edukativen Webseiten nachgewiesen werden. Till et al. (2017) fanden eine Reduktion an Suizidgedanken mittlerer Effektstär- 
Tab. 1 Übersicht über publizierte randomisierte, kontrollierte Studien zum Papageno-Effekt

\begin{tabular}{|c|c|c|c|c|}
\hline Quelle & Stichprobe & $\begin{array}{l}\text { Untersuchtes } \\
\text { Medium }\end{array}$ & Untersuchte Outcome-Variablen & Protektiver Effekt \\
\hline $\begin{array}{l}\text { Till et al. } \\
\text { (2015) }\end{array}$ & $\begin{array}{l}95 \text { Erwachsene } \\
\text { der allgemeinen } \\
\text { Bevölkerung }\end{array}$ & Hollywood-Film & $\begin{array}{l}\text { Suizidalität, Lebenszufriedenheit, Depressivität, } \\
\text { aktuelle Stimmung, Selbstwertgefühl, Einstellung zur } \\
\text { Welt }\end{array}$ & $\begin{array}{l}\text { Anstieg der Lebenszufriedenheit, positivere } \\
\text { Einstellung zur Welt }\end{array}$ \\
\hline $\begin{array}{l}\text { Arendt et al. } \\
\text { (2016) }\end{array}$ & 112 Studierende & Nachrichtenartikel & Suizidkognitionen & $\begin{array}{l}\text { Rückgang an Suizidkognitionen bei Teil- } \\
\text { nehmer_Innen mit geringer Identifikation mit } \\
\text { der geschilderten Problemsituation }\end{array}$ \\
\hline $\begin{array}{l}\text { Till et al. } \\
\text { (2017) }\end{array}$ & $\begin{array}{l}161 \text { Erwachsene } \\
\text { der allgemeinen } \\
\text { Bevölkerung }\end{array}$ & $\begin{array}{l}\text { Edukative Web- } \\
\text { seiten }\end{array}$ & $\begin{array}{l}\text { Primär: Suizidalität } \\
\text { Sekundär: Aktuelle Stimmung, Wissen über Sui- } \\
\text { zidprävention, Einstellung zu Suizid, Einstellung zu } \\
\text { professioneller Hilfe }\end{array}$ & $\begin{array}{l}\text { Primär: Reduktion an Suizidalität bei Teil- } \\
\text { nehmer_Innen mit vergleichsweise höherer } \\
\text { Suizidalität } \\
\text { Sekundär: Anstieg an Wissen über Suizidprä- } \\
\text { vention }\end{array}$ \\
\hline $\begin{array}{l}\text { Arendt et al. } \\
\text { (2018a) }\end{array}$ & $\begin{array}{l}273 \text { Erwachsene } \\
\text { der allgemeinen } \\
\text { Bevölkerung }\end{array}$ & Nachrichtenartikel & $\begin{array}{l}\text { Wissen über Suizidprävention, Hilfsbereitschaft, } \\
\text { Bewerung des Artikels, subjektiver Wissensgewinn }\end{array}$ & $\begin{array}{l}\text { Anstieg an Wissen über Suizidprävention und } \\
\text { Hilfsbereitschaft nach Lesen eines Artikels, } \\
\text { der auf Krisenbewältigung fokussiert und } \\
\text { Mythen über Suizid entkräftet }\end{array}$ \\
\hline $\begin{array}{l}\text { King et al. } \\
\text { (2018b, } \\
\text { 2018a) }\end{array}$ & $\begin{array}{l}337 \text { Erwachsene } \\
\text { Männer der } \\
\text { allgemeinen } \\
\text { Bevölkerung }\end{array}$ & $\begin{array}{l}\text { Dokumentations- } \\
\text { reihe }\end{array}$ & $\begin{array}{l}\text { Primär: Hilfesuchverhalten } \\
\text { Sekundär: Einstellung zu Hilfe, Konformität zu mas- } \\
\text { kulinen Normen, Erfahrung von Maskulinität, soziale } \\
\text { Unterstützung, Distress, Befindlichkeit, Resilienz, } \\
\text { Suizidalität, Bewertung der Serie }\end{array}$ & $\begin{array}{l}\text { Primär: Anstieg an Hilfesuchverhalten } \\
\text { Sekundär: Verbesserte Einstellung zu Hilfe, } \\
\text { Reduktion an Konformität zu maskulinen } \\
\text { Normen }\end{array}$ \\
\hline $\begin{array}{l}\text { Till et al. } \\
\text { (2019) }\end{array}$ & $\begin{array}{l}527 \text { Erwachsene } \\
\text { der allgemeinen } \\
\text { Bevölkerung }\end{array}$ & Nachrichtenartikel & $\begin{array}{l}\text { Primär: Suizidalität } \\
\text { Sekundär: Wissen über Suizidprävention }\end{array}$ & $\begin{array}{l}\text { Primär: Reduktion an Suizidalität } \\
\text { Sekundär: Anstieg an Wissen über Suizidprä- } \\
\text { vention }\end{array}$ \\
\hline
\end{tabular}

ke unter jenen User_Innen der Interventionsgruppe, die eine vergleichweise höhere Suizidalität aufwiesen, sowie einen starken Anstieg an Wissen über Suizidprävention bei allen User_Innen im Vergleich zur Kontrollgruppe. Beide Effekte konnten fast unvermindert auch noch eine Woche später festgestellt werden (Till et al. 2017).

Auch Onlineforen über Suizid gehören zu jenen Arten von Webseiten, die häufig vorzufinden sind, wenn man in Suchmaschinen nach suizidbezogener Information sucht (Till und Niederkrotenthaler 2014a). In einer Analyse von sieben deutschsprachigen Onlineforen über Suizid (Niederkrotenthaler et al. 2016) zeigte sich, dass es in nicht-professionellen Foren fallweise auch dazu kommt, dass jene User_Innen, die eine Diskussion starten, an Ende der Diskussion äußern, dass es ihnen nun besser geht als zuvor, sich also ihre Suizidalität, Hoffnungslosigkeit oder Überforderung mit ihren Problemen reduziert hat. Solche Anzeichen einer psychischen Besserung traten in ca. $10 \%$ der Diskussionen in sogenannten „ProSuizid-Foren“ und in knapp $45 \%$ der Diskussionen in „Anti-Suizid-Foren“ auf. „Anti-Suizid-Foren“ bezeichnen hier Foren, die u.a. auf Zusammenarbeit mit professionellen Hilfseinrichtungen Wert legen und grundsätzlich keine Methodendiskussionen auf ihren Seiten erlauben, während diese in sogenannten „ProSuizid-Foren“ sogar beworben werden. Es ist nicht verwunderlich, dass sich, in Anbetracht der Gefahren von „Pro-Suizid-Foren“, die Anzahl der Poster_Innen, die eine psychologische Verbesserung angaben, in Grenzen hielt (Niederkrotenthaler et al. 2016). Es ist allerdings erstaunlich, dass in allen Foren dieser positive Effekt mit bestimmten Reaktionen und Kommu- nikationsmustern der Respondent_Innen assoziiert war. Dazu gehörten u.a. Schilderungen vom Umgang mit eigenen Krisen und aktives Zuhören. Wenn also Respondent_Innen davon erzählt haben, wie sie selbst mit schweren Lebenssituationen umgehen oder in der Vergangenheit umgegangen sind, so schien es dem_der jeweiligen Erstposter_In danach besser zu gehen (Niederkrotenthaler et al. 2016). Auch dieser empirische Befund ist konsistent mit dem PapagenoEffekt und zeigt, dass der Fokus auf Krisenbewältigung in medialer Kommunikation über Suizidalität einen protektiven Effekt haben kann.

Alle der bisher diskutierten Studien analysierten den Papageno-Effekt anhand von Stichproben aus der allgemeinen Bevölkerung und nicht klinischen Propulationen oder Risikogruppen. In vielen der hier zitierten Studien wurden Personen, die ein erhöhtes Suizidrisiko aufwiesen, dezidiert ausgeschlossen (Till et al. 2019). Studien zum Papageno-Effekt mit vulnerablen Populationen gibt es bis jetzt noch kaum. Erste Ergebnisse deuten allerdings darauf hin, dass auch vulnerable Gruppen von Berichten über erfolgreiche Krisenbewältigungen profitieren (Niederkrotenthaler 2018): In einer Stichprobe bestehend aus Personen mit rezenten Suizidgedanken zeigte sich im Vergleich zur Kontrollgruppe ein Rückgang an Suizidalität nach dem Lesen eines Nachrichtenartikels, in dem eine Protagonistin schilderte, wie sie ihre suizidale Krise erfolgreich bewältigt hatte. Dieser positive Effekt zeigte sich insbesondere bei jenen Proband_Innen, die im letzten Jahr einen Suizidversuch unternommen hatten. Die Geschichte hatte jedoch keinen signifikanten positiven Effekt, wenn sie nicht von der selbst betroffenen Person, sondern von einer Expertin für 
Suizidprävention erzählt wurde (Niederkrotenthaler und Till 2019). In einer weiteren Studie sahen junge Erwachsene mit akuten Symptomen einer Depression und Suizidgedanken entweder ein Video, in dem eine junge Frau von ihrem Kampf gegen Depressionen und Suizidgedanken und ihrer erfolgreichen Behandlung erzählte oder ein Kontrollvideo ohne Bezug zu Suizid oder psychischer Gesundheit. Die Teilnehmer_Innen, die das Video über Depression sahen, berichteten von einem Rückgang ihrer depressiven Symptomatik. In der Kontrollgruppe konnte hingegen ein Anstieg an Suizidalität bei jenen Teilnehmer_Innen festgestellt werden, die vergleichsweise stärkere Symptome von Depressionen aufwiesen, wohingegen sich in der Interventionsgruppe kein solcher Effekt zeigte (Niederkrotenthaler und Till 2020). Insgesamt deuten diese Befunde darauf hin, dass auch Gruppen von vulnerablen Personen mit einem Rückgang des Suizidrisikos reagieren, wenn sie mediale Darstellungen von erfolgreichen Krisenbewältigungen rezipieren. Eine zusammenfassende Übersicht über alle bisher durchgeführten randomisierten, kontrollierten Studien zum Papageno-Effekt befindet sich in Tab. 1.

\section{Konklusion}

Die Ergebnisse der hier diskutierten Studien zeigen, dass Suiziddarstellungen in den Medien das suizidale Verhalten der Rezipient_Innen beeinflussen können. Während sensationsträchtige Darstellungen von Suizid und der Fokus auf Suizidmethoden einen schädlichen Effekt haben und zu Imitationssuiziden führen (Werther-Effekt), können Geschichten über Menschen, die in der Lage waren, ihre suizidalen Krisen erfolgreich zu bewältigen, einen Rückgang an Suizidalität bei den Rezipient_Innen bewirken (PapagenoEffekt). Die Effekte von Suiziddarstellungen hängen davon ab, auf welche Aspekte von Suizidalität fokussiert wird und wer diese Darstellungen rezipiert. Erkenntnisse zum Papageno-Effekt sind für die Suizidprävention von großem Wert, da sie Aufschluss darüber geben können, wie Medienkampagnen zur Aufklärung über Suizid gestaltet werden müssen, um eine möglichst positiven Effekt $\mathrm{zu}$ erzielen, ohne dabei etwaige Imitationseffekte auszulösen. Geschichten von Menschen, die sich in suizidalen Krisen befunden haben, sich aber Hilfe gesucht und dadurch die Krise bewältigt haben, können hier offenbar einen wichtigen Beitrag leisten. Dementsprechend gibt es auch seit einigen Jahren zunehmend Bestrebungen, Menschen mit persönlicher Erfahrung mit Suizidalität mehr in Strategien und in der Entwicklung von Ressourcen der Suizidprävention einzubeziehen (National Action Alliance for Suicide Prevention 2014; Till et al. 2019). Diese Idee ist an und für sich keineswegs neu. In anderen medizinischen Bereichen der Aufklärung, wie etwa bei der Prävention von Krebs oder Herzkreislauferkrankungen, ist es schon seit Jahren gängige Praxis, nicht alleine Mortalitätsraten zu be- richten, sondern dabei verschiedenste Therapiemöglichkeiten und Behandlungserfolge zu erwähnen. Daher sollten auch in der Berichterstattung über Suizidalität Behandlungserfolge und Möglichkeiten für Auswege aus der Krise Eingang finden, um Suizide aktiv zu verhüten (Till und Niederkrotenthaler 2014b). Auch könnte die Integration medialer oder literarischer Darstellungen von Krisenbewältigungen in psychotherapeutische Gespräche Patient_Innen bei der Bewältigung aktueller suizidaler Krisen unterstützen. So werden zum Beispiel in der Kinotherapie bereits seit geraumer Zeit Filme eingesetzt, um sowohl bei ambulanten als auch stationären Patient_Innen mit Suchterkrankungen Hoffnung auf eine gelingende Lebensveränderung zu induzieren (Poltrum 2009; Poltrum und Leitner 2009). Hierbei ist aber auch die Forschung gefragt, die genauen Mechanismen des Papageno-Effekts näher zu erforschen, insbesondere in Studien mit Risikogruppen für Suizid.

Danksagung Die hier präsentierten Forschungsergebnisse zu Online-Medien wurden durch den FWF gefördert (GrantNr. P-23659-B11). Teile dieses Artikels wurden in abgeänderter Form und in englischer Sprache in De Leo \& Postuvan (Eds.) (2019), Reducing the Toll of Suicide: Resources for Communities, Groups, and Individuals. Hogrefe Publishing. www. hogrefe.com, in Niederkrotenthaler \& Stack (Eds.) (2017), Media \& suicide: International perspectives on research, theory, \& policy. Transaction Books, sowie in der Zeitschrift Suicidologi (https://www.med.uio.no/klinmed/english/research/ centres/nssf/resources/the-journal-suicidologi/) (Niederkrotenthaler und Till, in Druck) veröffentlicht.

Funding Open access funding provided by Medical University of Vienna.

Interessenkonflikt B. Till und T. Niederkrotenthaler geben an, dass kein Interessenkonflikt besteht.

Open Access Dieser Artikel wird unter der Creative Commons Namensnennung 4.0 International Lizenz (http:// creativecommons.org/licenses/by/4.0/deed.de) veröffentlicht, welche die Nutzung, Vervielfältigung, Bearbeitung, Verbreitung und Wiedergabe in jeglichem Medium und Format erlaubt, sofern Sie den/die ursprünglichen Autor(en) und die Quelle ordnungsgemäß nennen, einen Link zur Creative Commons Lizenz beifügen und angeben, ob Änderungen vorgenommen wurden.

\section{Literatur}

Arendt, F., Scherr, S., Niederkrotenthaler, T., Krallmann, S., \& Till, B. (2018a). Effects of awareness material on suiciderelated knowledge and the intention to provide adequate help to suicidal individuals. Crisis, 39, 47-54.

Arendt, F., Scherr, S., Niederkrotenthaler, T., \& Till, B. (2018b). The role of language in suicide reporting: Investigating the influence of problematic suicide referents. Social Science \& Medicine, 208, 165-171.

Arendt, F., Scherr, S., Till, B., Prinzellner, Y., Hines, K., \& Niederkrotenthaler, T. (2017). Suicide on TV: minimising the risk to vulnerable viewers. $B M J, 358$, j3876. https://doi. org/10.1136/bmj.j3876. 
Arendt, F, Till, B., \& Niederkrotenthaler, T. (2016). Effects of suicide awareness material on implicit suicide cognition: A laboratory experiment. Health Communication, 31, $718-726$.

Bandura, A. (1977). Social learning theory. Englewood Cliffs: Prentice-Hall.

Blood, R. W., \& Pirkis, J. (2001). Suicide and the media. Part III: Theoretical issues. Crisis, 22, 163-169.

Bridge, J.A., Greenhouse, J. B., Ruch, D., Stevens, J., Ackerman, J., Sheftall, A.H., et al. (2019). Association between the release of Netflix's 13 Reasons Why and suicide rates in the United States: An interrupted times series analysis. Journal of the American Acadademy of Child and Adolescent Psychiatry..https://doi.org/10.1016/j.jaac.2019.04.020.

Cooper, M.T., Bard, D., Wallace, R., Gillapsy, S., \& Deleon, S. (2018). Suicide attempt admissions from a single children's hospital before and after the introduction of Netflix series 13 Reasons Why. Journal of Adolescent Health, 63, 688-693.

De Leo, D., \& Postuvan, V. (Hrsg.). (2019). Reducing the toll of suicide: Resources for communities, groups, and individuals. Boston: Hogrefe.

Etzersdorfer, E., \& Sonneck, G. (1998). Preventing suicide by influencing mass-media reporting. The Viennese experience 1980-1996. Archives of Suicide Research, 4(1), 67-74. https://doi.org/10.1080/13811119808258290.

Etzersdorfer, E., Sonneck, G., \& Nagel Kuess, S. (1992). Newspaper reports and suicide. New England Journal of Medicine, 327, 502-503.

Fink, D. S., Santaella-Tenorio, J., \& Keyes, K. M. (2018). Increase in suicides the months after the death of Robin Williams in the US. PLoS ONE, 13, e191405.

Fu, K.W., \& Yip, P.S. (2009). Estimating the risk for suicide following the suicide deaths of three Asian entertainment celebrities: a meta-analysis approach. Journal of Clinical Psychiatry, 70, 869-878.

Gould, M.S., \& Shaffer, D. (1986). The impact of suicide in television movies: Evidence of imitation. New England Journal of Medicine, 315, 690-694.

Hawton, K., \& Williams, K. (2002). Influences of the media on suicide. Researchers, policy makers, and media personnel need to collaborate on guidelines. British Medical Journal, $325,1374-1375$

Jetter, M. (2017). The effect of media attention on terrorism. Journal of Public Economics, 153,32-48.

Kahneman, D. (2011). Thinking, fast and slow. London: Penguin.

King, K. E., Schlichthorst, M., Reifels, L., Keogh, L., Spittal, M. J., Phelps, A., \& Pirkis, J. (2018a). Impacts of a documentary about masculinity and men's health. American Journal of Men's Health, 12, 1604-1614.

King, K.E., Schlichthorst, M., Spittal, M. J., Phelps, A., \& Pirkis, J. (2018b). Can a documentary increase help-seeking intentions in men? A randomised controlled trial. Epidemiology, 72, 92-98.

Mann, J. J., Apter, A., Bertolote, J., Beautrais, A., Currie, D., Haas, A., et al. (2005). Suicide prevention strategies-a systematic review. JAMA, 294, 2064-2074.

Michel, K., Frey, C., Wyss, K., \& Valach, L. (2000). An exercise in improving suicide reporting in print media. Crisis, 21, $71-79$

Mindframe (2007). Mental illness and suicide. A Mindframe resource for stage and screen. Canberra: Commonwealth of Australia.

NationalActionAlliancefor SuicidePrevention (2014). Theway forward: Pathways to hope, recovery, and wellness with insights from lived experiences. Washington, DC:National Action Alliance for Suicide Prevention.

National Action Alliance for Suicide Prevention (2019). National recommendations for depicting suicide. https:// theactionalliance.org/messaging/entertainmentmessaging/national-recommendations. Zugegriffen: 13. März2019

Niederkrotenthaler, T. (2018). Media ands suicide: From Werther to Papageno effects. In 18th European Symposium of Suicide and Suicidal Behaviour. Ghent, Belgium, September 5-8, 2018. Oral presentation.

Niederkrotenthaler, T., \& Sonneck, G. (2007). Assessing the impact of media guidelines for reporting on suicides in Austria: interrupted times series analysis. Australian and New Zealand Journal of Psychiatry, 41,419-428.

Niederkrotenthaler, T., \& Stack, S. (Hrsg.). (2017). Media \& suicide: international perspectives on research, theory, \& policy. Piscataway: Transaction Books.

Niederkrotenthaler, T., \&Till, B. (2019). Effects of suicide awareness materials on individuals with recent suicidal ideation or attempt: Randomized controlled online trial. Unveröffentlichtes Manuskript

Niederkrotenthaler, T., \& Till, B. (2020). Effects of awareness material featuring individuals with experience of depression and suicidal thoughts on an audience with depressive symptoms: Randomized controlled trial. Journal of Behavior Therapy \& Experimental Psychiatry, 66, 101515. https://doi.org/10.1016/j.jbtep.2019.101515.

Niederkrotenthaler, T., \& Till, B. (in Druck). Suicide and the media: From Werther to Papageno effects-A selective literature review. Suicidologi.

Niederkrotenthaler, T., Voracek, M., Herberth, A., Till, B., Strauss, M., Etzersdorfer, E., et al. (2010a). The role of media reports in completed and prevented suicide-Werther versus Papageno effects. British Journal of Psychiatry, 197, 234-243.

Niederkrotenthaler, T., Voracek, M., Herberth, A., Till, B., Strauss, M., Etzersdorfer, E., et al. (2010b). Papageno v Werther effect. British Medical Journal, 341,5841.

Niederkrotenthaler, T., Gould, M., Sonneck, G., Stack, S., \& Till, B. (2016). Predictors of psychological improvement on non-professional suicide message boards: Content analysis. Psychological Medicine, 46, 3429-3442.

Niederkrotenthaler, T., Herberth, A., \& Sonneck, G. (2007). Der Werther Effekt: Mythos oder Realität? Neuropsychiatrie, 21,235-243.

Niederkrotenthaler, T., Reidenberg, D. J., Till, B., \& Gould, M. (2014). Increasing help-seeking and referrals for individuals at risk for suicide by decreasing stigma: The role of mass media. American Journal of Preventive Medicine, 47, 235-243.

Niederkrotenthaler, T., Stack, S., Till, B., Sinyor, M., Pirkis, J., Garcia, D., Rockett, I. R. H., \& Tran, U.S. (2019). Suicides in the United States after the release of 13 Reasons Why: Time series analysis. JAMA Psychiatry, 76, 933-940.

Niederkrotenthaler, T., Till, B., Kapusta, N.D., Voracek, M., Dervic, K., \& Sonneck, G. (2009). Copycat effects after media reports on suicide: A population-based ecologic study. Social Science \& Medicine, 69, 1085-1090.

Nock, M., Park, J., Finn, C., Deliberto, T., Dour, H., \& Banaji, M. (2010). Measuring the suicidal mind: Implicit cognition predicts suicidal behavior. Psychological Science, 21, 511-517.

Phillips, D. P. (1974). The influence of suggestion on suicide: Substantive and theoretical implications of the Werther effect. American Sociological Review, 39, 340-354. 
Pirkis, J., \& Blood, W. (2001a). Suicide and the media. Part I: Reportage in nonfictionalmedia. Crisis, 22, 146-154.

Pirkis, J., \& Blood, W. (2001b). Suicide and the media. Part II: Portrayal in fictional media. Crisis, 22, 155-162.

Pirkis, J., Blood, R.W., Beautrais, A., Burgess, P., \& Skehan, J. (2006).Mediaguidelines on thereporting of suicide. Crisis, 27, 82-87.

Pirkis, J., Dare, A.R., Blood, W., Rankin, B., Williamson, M., Burgess, B., \& Jolley, D. (2009). Changes in media reporting of suicide in Australia between 2000/01 and 2006/07. Crisis, 30, 25-33.

Poltrum, M. (2009). Cinematherapy in der Suchtbehandlung: Filme zur Induktion positiver Veränderungserwartung. Spectrum Psychiatrie, 4, 18-20.

Poltrum, M., \& Leitner, R. (2009). Existenzanalytische Kinotherapie Filme als Balsam der Seele. Wiener Zeitschrift für Suchtforschung, 32, 47-52.

Pouliot, L., Mishara, B. L., \& Iabelle, R. (2011). The Werther effect reconsidered in light of psychological vulnerabilities: Results of a pilot study. Journal of Affective Disorders, 134, 488-496.

Ringel, E. (1976). The presuicidal syndrome. Suicide and LifeThreatening Behavior, 6, 131-149.

Samaritans (2017). Media guidelines: Factsheet drama portrayals. https://www.samaritans.org/sites/default/files/ kcfinder/files/press/Samaritans\%20media\%20factsheet \%20-\%20drama\%20portrayal.pdf. Zugegriffen: 29. Dezember 2018

Schlichthorst, M., King, K.E., Spittal, M. J., Reifels, L., Phelps, A., \& Pirkis, J. (2018). Using a television documentary to prevent suicide in men and boys. Australasian Psychiatry, $26,160-165$

Schmidtke, A., \&Häfner, H. (1986). Die Vermittlung von Selbstmordmotivation und Selbstmordhandlung durch fiktive Modelle. Die Folgen der Fernsehserie „Tod eines Schülers". Nervenarzt, 57, 502-510.

Schütte, C. (2008). Selbsttötung als Spektakel? Suiziddarstellungen in Boulevardzeitungen. In A. Herberth, T. Niederkotenthaler \& B. Till (Hrsg.), Suizidalität in den Medien/Suicidality in the media: Interdisziplinäre Betrachtungen/Interdisciplinary contributions(S.241-259). Münster: LIT.

Sisask, M., \& Värnik, A. (2012). Media roles in suicides prevention: A systematic review. International Journal of Environmental Research and Public Health, 9, 123-138.

Stack, S. (1992). Social correlates of suicide by age. Media impacts. In A. A. Leenaars (Hrsg.), Life span perspectives of suicide: Time lines in the suicidal process (S. 187-214). NewYork: Plenum.
Suicide Awareness Voices of Education (2017). Recommendations for reporting on mass shootings. https://www. reportingonmassshootings.org/. Zugegriffen: 22. Mai 2019

Till, B., \& Niederkrotenthaler, T. (2014a). Surfing for suicide methods and help: Content analysis of websites retrieved with search engines in Austria and in the United States. Journal of Clinical Psychiatry, 75, 886-892.

Till, B., \& Niederkrotenthaler, T. (2014b). Vom Werther- zum Papageno-Effekt. Die Rollen von Medien in der Suizidprävention. JATROS - Neurologie \& Psychiatrie, 6, 27-30.

Till, B., \& Niederkrotenthaler, T. (2018). Werther- und Papageno-Effekt durch Suiziddarstellungen im Internet Suizidgefahr durch neue Medien? Spectrum Psychiatrie, 3 , 26-29.

Till, B., Arendt, F., Scherr, S., \& Niederkrotenthaler, T. (2019). Effect of educative suicide prevention news articles featuring experts with vs without personal experience of suicidal ideation: A randomized controlled trial of the $\mathrm{Pa}$ pageno effect. Journal of Clinical Psychiatry. https://doi. org/10.4088/jcp.17m11975.

Till, B., Niederkrotenthaler, T., Herberth, A., Vitouch, P., \& Sonneck, G. (2010). Suicide in films: The impact of suicide portrayals on non-suicidal viewers' well-being and the effectiveness of censorship. Suicide \& Life-Threatening Behavior, 40,319-327.

Till, B., Strauss, M., Sonneck, G., \&Niederkrotenthaler,T. (2015). Determining the effects of films with suicidal content: a laboratory experiment. British Journal of Psychiatry, 207,72-78.

Till, B., Tran, U. S., Voracek, M., \& Niederkrotenthaler, T. (2017). Papageno vs. Werther effect online: Randomized controlled trial of beneficial and harmful impacts of educative suicide prevention websites. British Journal of Psychiatry, $211,109-115$.

Till, B., Wild, T. A., Arendt, F., Scherr, S., \& Niederkrotenthaler, T. (2018). Associations of tabloid newspaper use with endorsement of suicide myths, suicide-related knowledge, and stigmatizing attitudes toward suicidal individuals. Crisis, $39,428-437$.

Tomandl, G., Sonneck, G., Stein, C., \& Niederkrotenthaler, T. (2019). Leitfaden zur Berichterstattung über Suizid.Wien: Kriseninterventionszentrum.

World Health Organization (2017). Preventing suicide. A resource for media professionals-Update 2017. Genf: World Health Organization.

Hinweis des Verlags Der Verlag bleibt in Hinblick auf geografische Zuordnungen und Gebietsbezeichnungen in veröffentlichten Karten und Institutsadressen neutral. 